\title{
RESOLUTIONS OF SINGULARITIES IN PRIME CHARACTERISTIC FOR ALMOST ALL PRIMES( $\left.{ }^{1}\right)$
}

BY

PAUL C. EKLOF $\left({ }^{2}\right)$

0. Introduction. The existence of resolutions of singularities of algebraic varieties defined over fields of characteristic zero has been proved by Hironaka [2]; for algebraic varieties defined over fields of characteristic $p \neq 0$, the existence of resolutions is known only for varieties of dimension 2 (for all $p$ ) and dimension 3 (for $p \neq 2,3,5$ ). In this paper we use Hironaka's Theorem and techniques of ultraproducts to obtain a partial answer to this question, namely a result which (roughly) asserts the existence for all but a finite number of primes of resolutions of singularities of varieties defined over fields of prime characteristic, where the exceptional set of primes depends on certain numerical parameters of the varieties. In particular, we prove the following (where "nonsingular" means "smooth" in Grothendieck's terminology)

TheORem A. For any pair $(n, d)$ of positive integers there exists a finite set $P_{0}(n, d)$ of primes, and positive integers $t^{\prime}, n^{\prime}, d^{\prime}$ such that if $X$ is any projective F-variety satisfying:

(1) characteristic of $F$ is not in $P_{0}(n, d)$; and

(2) $X$ can be embedded in projective $n$-space, as a subvariety of degree $d$;

then there exists a finite sequence of monoidal transformations of F-varieties $\pi_{l}: X_{l+1} \rightarrow X_{l}, 0 \leqq l<t$, such that $t \leqq t^{\prime}, X_{0}=X, X_{t}$ is nonsingular, and satisfying for all l:

(3) the center of $\pi_{l}$, say $Y_{l}$, is nonsingular;

(4) $Y_{l}$ contains no nonsingular points of $X_{l}$; and

(5) $X_{l}$ can be embedded in projective $n^{\prime}$-space as a subvariety of degree $\leqq d^{\prime}$ $(l=0, \ldots, t)$.

Condition (1) is satisfied if Char $F=0$. In that case, the existence of the resolution is Hironaka's Theorem-which is used in the proof of Theorem A-but we also assert the existence of the bounds $t^{\prime}, n^{\prime}$, and $d^{\prime}$. (We thank Abraham Robinson for calling to our attention this application of our methods to characteristic zero.)

Presented to the Society, January 23, 1969, received by the editors March 6, 1969 and, in revised form, June 22, 1969.

( $\left.{ }^{1}\right)$ This paper contains the principal results of the author's Ph.D. thesis (Cornell University 1968). I wish to thank Professor James Ax for his advice and encouragement.

$\left({ }^{2}\right)$ Research partially supported by an NSF Graduate Fellowship and by the Air Force Office of Scientific Research, Office of Aerospace Research, United States Air Force, under AFOSR Contract No. F 44620-67-C-0090. 
(Here and throughout this paper the geometric terms are defined as in Weil [12] or Lang [7] unless otherwise specified. We recall that, by definition, an $F$-variety is irreducible over $F$ but not necessarily absolutely irreducible, and nonsingularity means the Jacobian criterion is satisfied, which in finite characteristic is a stronger condition than regularity. We review the definition of monoidal transformation in \$4.)

We remark that there are projective $F$-varieties which are not birationally equivalent to any nonsingular $F$-variety (see [13]). In one sense therefore Theorem $\mathrm{A}$ is a strongest possible result. But, of course, it is not known if every projective variety is birationally equivalent to a regular variety, and in this sense the resolution of singularities may hold for all primes.

We also obtain a true theorem-which we call Theorem $A^{\prime}-$ if we replace (2) by:

The maximum of the dimensions of the Zariski tangent spaces to $V$ at points of $V=n$; and the minimal embedding degree of $V=d$;

and if we also change (5) analogously. Moreover in Theorem $\mathrm{A}^{\prime}$ we require $F$ to be infinite. Theorem $\mathrm{A}^{\prime}$ follows from Theorem $\mathrm{A}$ by an application of the embedding theorem for projective varieties [8].

The most general-but least geometric-formulation of our result is Theorem 4.2 of $\$ 4$. Theorem 4.2 is stated for arbitrary abstract varieties and involves the parameter of bounded type, which is introduced in $\S 1$. In $\$ \S 2-4$ we define and study the properties of the algebraic construction which is the basis of our proof, namely, the ultraproduct of a family of varieties of bounded type. In $\$ 4$ we prove Theorem 4.2 and derive Theorem $\mathrm{A}$ from it by interpreting the parameter of bounded type in more geometric terms.

Finally we remark that the proof we give of Theorem 4.2 is purely algebraic, using ultraproducts, but we could also give another version using the Compactness Theorem of logic; in that version our auxiliary results about relations between certain properties of ultraproducts and of their components would be translated into results that assert that certain properties are expressible as elementary statements in the language of fields.

1. Ideals and varieties of bounded type. Throughout the paper $I$ denotes an arbitrary (index) set and $i$ an element of $I$. The indexing of a collection by $I$ employs the superscript $i . F$ and $F^{i}$ will always denote fields.

1.1 Definition. An ideal $A \subseteq F\left[X_{1}, \ldots, X_{n}\right]$ is of bounded type $M$ if $n \leqq M$ and $A$ has a basis of polynomials of (total) degree $\leqq M$. A family $\left\{A^{i} \mid i \in I\right\}$ of polynomial ideals is of bounded type if there is a positive integer $M$ such that $A^{i}$ is of bounded type $M$ for each $i \in I$.

(Obviously, if $A$ is of bounded type $M$, then $A$ is of bounded type $N$ for any $N \geqq M$. We could associate a unique integer with $A$, namely the minimal $M$ such that $A$ is of bounded type $M$, but this is of no particular advantage to us.) 
Note that for any $M$ there is a positive integer $\alpha(M)$ such that if $A$ is of bounded type $M, A$ has a basis of cardinality $\leqq \alpha(M)$ consisting of polynomials of degree $\leqq M$.

1.2 Definition. Let $X$ be an affine (respectively projective) $F$-variety embedded in affine (resp. projective) $n$-space, with (radical) ideal $A \subseteq F\left[X_{1}, \ldots, X_{n}\right]$ (resp. $\left.A \subseteq F\left[X_{0}, \ldots, X_{n}\right]\right)$. Then $X$ is said to be of bounded type $M$ if $A$ is of bounded type $M$. A family $\left\{X^{i} \mid i \in I\right\}$ of varieties is of bounded type if there exists $M$ such that $X^{i}$ is of bounded type $M$ for every $i \in I$.

Before proving a theorem which will give a geometric interpretation of the concept of bounded type we state a result of G. Hermann [1] which we will have occasion to use more than once in this paper. $\left(Z_{>0}=\right.$ positive integers.)

1.3 TheOREM. For any $q, n \in Z_{>0}$ there exists $m(q, n) \in Z_{>0}$ such that if $F$ is any field and $A$ is any ideal in $F\left[X_{1}, \ldots, X_{n}\right]$ generated by polynomials of degree $\leqq q$, then the radical $\sqrt{ } A$ of $A$ is generated by polynomials of degree $\leqq m(q, n)$.

(Hermann shows how, given the ideal $A$, to construct "in a finite number of steps" a set of generators for $\sqrt{ } A$. An analysis of Hermann's proof demonstrates the existence of the bound $m(q, n)$. See also Kleiman [3] for another proof.)

1.4 TheOREM. For each $i \in I$, let $X^{i}$ be an $F^{i}$-variety embedded in projective $n^{i}$-space as a subvariety of degree $\leqq d^{i}$. Then $\left\{X^{i} \mid i \in I\right\}$ is of bounded type $\Leftrightarrow$ there exists $N \in Z_{>0}$ such that for each $i \in I, n^{i} \leqq N$ and $d^{i} \leqq N$.

Proof. Suppose that $X^{i}$ is of bounded type $n$ for every $i \in I$; so $n^{i} \leqq n$ and we may assume $X^{i} \subseteq P^{n}$, for all $i \in l$. Now if $r^{i}=\operatorname{dim} X^{i}(\leqq n)$ and the ideal of $X^{i}$ is generated by polynomials $f_{1}\left(Y_{0}, \ldots, Y_{n}\right), \ldots, f_{s}\left(Y_{0}, \ldots, Y_{n}\right)$, then $d^{i}\left(r^{i}+1\right)$ $=$ degree of the defining equation $\psi$ of the hyperplane $S \subseteq P^{m}\left(m=n\left(r^{i}+1\right)\right)$, where $S$ is the projection on the second factor of the subvariety of $P^{n} \times P^{m}$ defined by the equations:

$$
\begin{aligned}
f_{k}\left(Y_{0}, \ldots, Y_{n}\right) & =0, & k & =1, \ldots, s, \\
\sum_{l=0}^{n} X_{j l} Y_{l} & =0, & j & =0, \ldots, r^{i} .
\end{aligned}
$$

(See van der Waerden [11, pp. 156f].) Thus $\psi(X)$ may be computed from this system of equations by eliminating the variables $Y_{0}, \ldots, Y_{n}$ and hence there is an integer $n^{\prime}$ depending only on $n$ such that $\operatorname{deg} \psi(X) \leqq n^{\prime}$. So let $N \geqq \max \left\{n, n^{\prime} / n\right\}$.

Conversely suppose $n^{i}$ and $d^{i}$ are bounded above by $N$ for all $i$. Then an inspection of the procedure in van der Waerden $[11, \S 37]$ for obtaining a set of equations for $X^{i}$ from the associated form for $X^{i}$, shows that the set of equations is bounded in degree by a function of $n^{i}$ and $d^{i}$. This set of equations may not generate the radical ideal of $X^{i}$, but we can apply Theorem 1.3 to conclude the proof.

2. Ultraproducts of affine and projective varieties. Let $F^{t}, i \in I$, be a family of fields. For a fixed ultrafilter $D$ on $I$, let $F^{*}$ denote the ultraproduct, $\prod_{i \in I} F^{i} / D$, of 
the $F^{i}$ with respect to $D$. (For the definition and properties of ultraproducts, see for example Kochen [4].) Given families of polynomials, ideals, or varieties defined over the $F^{i}$, and of bounded type, we shall define their ultraproducts, which will be defined over $F^{*}$.

2.1 Definition. Suppose that $f^{i} \in F^{i}\left[X_{1}, \ldots, X_{n^{i}}\right]$ such that there exists $n \in Z_{>0}$ with $n^{i} \leqq n$ and $\operatorname{deg} f^{i} \leqq n$ for all $i \in I$. Write

$$
f^{i}=\sum_{(j) \in J} c_{(j,}^{i} X_{1}^{j_{1}} X_{2}^{j_{2}} \cdots X_{n^{n}}^{j_{n}}=\sum c_{(j)}^{i} X^{(j)}
$$

where $J=\left\{(j)=\left(j_{1}, \ldots, j_{n}\right) \mid j_{k} \in Z_{\geqq 0} \forall k ; \sum_{k=1}^{n} j_{k} \leqq n\right\}$.

Then the ultraproduct of the $f^{i}$ with respect to $D$, denoted $f^{*}$, or more explicitly $\left(f^{i}\right)_{D}^{*}$, is defined to be the polynomial

$$
f^{*}=\sum_{(j) \in J} c_{(j)}^{*} X_{1}^{j} X_{2}^{j} \cdots X_{n^{n}}^{j_{n}}=\sum c_{(j)}^{*} X^{(j)}
$$

where $c_{(j)}^{*}$ is the element of $F^{*}$ represented by $\left(c_{(j)}^{i}\right) \in \prod_{i \in I} F^{i}$.

2.2. It follows immediately from the definition of the ultraproducts of fields that if $x_{1}^{i}, \ldots, x_{n}^{i}$ are elements of $F^{i}$ and $x_{j}^{*}=$ the image of $\left(x_{j}^{i}\right)$ in $F^{*}$, then $f^{*}\left(x_{1}^{*}, \ldots, x_{n}^{*}\right)=0 \Leftrightarrow f^{i}\left(x_{1}^{i}, \ldots, x_{n}^{i}\right)=0$ for $i$ in a set of $D$.

(When a property such as the above holds for $i$ in a set of $D$ we shall say that it holds "for almost all $i$ (w.r.t.D)" or "almost everywhere (w.r.t.D)". This language is inspired by the correspondence between ultrafilters and finitely-additive measures on $I$ which take only the values 0 and 1 (cf. $[4, \S 3])$.)

2.3 Definition. If $A^{i} \subseteq F^{i}\left[X_{1}, \ldots, X_{n^{i}}\right], i \in I$, is a family of ideals of bounded type, then there exists a unique $n \in Z_{>0}$ such that $n^{i}=n$ for all $i$ in some set $S \in D$. Then we define the ultraproduct of the ideals $A^{i}$ with respect to $D$ to be the ideal $A^{*}=\left(A^{i}\right)_{D}^{*} \subseteq F^{*}\left[X_{1}, \ldots, X_{n}\right]$ which is generated by the ultraproducts, $f_{1}^{*}, \ldots, f_{\mathrm{r}}^{*}$ of a set of generators, $f_{1}^{i}, \ldots, f_{r}^{i}$ of the $A^{i}, i \in S$.

Note that by the definition of bounded type we may choose the generators $f_{1}^{i}, \ldots, f_{r}^{i}$ to be of bounded degree and cardinality for all $i \in S$. The proof that $A^{*}$ is well defined is a consequence of the observation that any relation of the type $g=\sum_{j=1}^{r} h_{j} f_{j}$ may be regarded as a finite set of relations on the coefficients of the polynomials $g, h_{j}$, and $f_{j}$, and of the following fact due to J. König ([5]; see also Hermann [1, p. 750]).

2.4 THEOREM. For any $t, q, n \in Z_{>0}$ there exists $m_{1}(t, q, n) \in Z_{>0}$ such that if $F$ is any field and $g, f_{1}, \ldots, f_{t}$ are polynomials in $F\left[X_{1}, \ldots, X_{n}\right]$ with $\operatorname{deg} f_{j}(X) \leqq q$, $\forall j=1, \ldots, t$ and $g \in\left\langle f_{1}, \ldots, f_{t}\right\rangle$, then there exist polynomials $h_{1}, \ldots, h_{t}$ $\in F\left[X_{1}, \ldots, X_{n}\right]$ such that $\operatorname{deg} h_{j} \leqq \operatorname{deg} g+m_{1}(t, q, n)$ and $g=\sum_{j=1}^{t} h_{j} f_{j}$.

Using 2.4 we can also prove the following

2.5 THEOREM. If $h^{i}, i \in I$, are polynomials of bounded degree and $h^{*}$ is their ultraproduct w.r.t.D, then $h^{*} \in A^{*} \Leftrightarrow h^{i} \in A^{i}$ for almost all $i$ (w.r.t.D). 
REMARK. $A^{*}$ is not in general isomorphic to the ultraproduct of rings $\prod_{i \in I} A^{i} / D$. (Indeed the latter may contain an infinite number of elements algebraically independent over $F^{*}$.) But if we let $R^{*}=\prod_{i \in I} F^{i}\left[X_{1}, \ldots, X_{n}\right] / D$, there is a canonical embedding $\rho: F^{*}\left[X_{1}, \ldots, X_{n}\right] \rightarrow R^{*}$ such that if $f^{t}$ and $f^{*}$ are as in 2.1 , then $\rho\left(f^{*}\right)=$ the element of $R^{*}$ represented by $\left(f^{i}\right)$; under this embedding $\rho\left(A^{*}\right)=$ $\left(\prod_{i \in I} A^{i} / D\right) \cap \rho\left(F^{*}\left[X_{1}, \ldots, X_{n}\right]\right)$, where $\prod_{i \in I} A^{i} / D$ is identified with a subring of $R^{*}$ in the obvious way.

2.6 THEOREM. Let $A^{i}, A^{*}$ be as in 2.3. Then $A^{*}$ is a radical ideal (respectively: has prime radical; is prime) $\Leftrightarrow A^{i}$ is radical (resp. has prime radical; is prime) for almost all $i$ (w.r.t.D).

Proof. The result for radical ideals is a consequence of $1.3,2.5$, and the following result of A. Robinson: for any $n \in Z_{>0}$ there exists $m=m(n) \in Z_{>0}$ such that if $A$ is a polynomial ideal of bounded type $n$, and $f \in \sqrt{ } A$ and $\operatorname{deg} f \leqq n$, then $f^{m} \in A$ $[9$, p. 127]. The result for ideals with prime radical is an immediate consequence of A. Robinson's proof that "prime radical" is elementarily definable [10]. (Robinson's proof is based on van der Waerden's method $[11 ; \S 31]$ for effectively decomposing a variety into its irreducible components.) The result for prime ideals is a consequence of the first two results or of W. Lambert's proof [6] that "prime" is elementarily definable (which in turn is based on Hermann's algebraic results).

2.7 Definition. Let $X^{i}, i \in I$, be a family of algebraic sets defined over fields $F^{i}$ and embedded in affine or projective space and of bounded type. Let $A^{i}$ be the ideal of $X^{i}$ over $F^{i}$, so that the $A^{i}$ are of bounded type. Then the ultraproduct of the $X^{i}$ with respect to $D$ is defined to be the algebraic set defined over $F^{*}$ by the ideal $A^{*}$; it is denoted $X^{*}$ or, more explicitly, $\left(X^{i}\right)_{D}^{*}$.

Note that by $2.6 A^{*}$ is a radical ideal so that it is in fact the ideal of an algebraic set. Also by $2.6, X^{*}$ is irreducible if and only if $X^{i}$ is irreducible for almost all $i$ (w.r.t.D).

(Open Question: If $\left\{Y^{i} \mid i \in I\right\}$ is of bounded type and $X^{i} \cong Y^{i}$ for almost all $i$ (w.r.t.D), is $X^{*} \cong Y^{*}$ ?)

2.8 TheOREM. (1) If $X^{*}$ is irreducible, $\operatorname{dim} X^{*}=\operatorname{dim} X^{i}$ for almost all $i$ (w.r.t.D); (2) $X^{*}$ is nonsingular $\Leftrightarrow X^{i}$ is nonsingular for almost all $i$ (w.r.t.D).

Proof. We may suppose $X^{i}$ is affine for all $i \in I$.

(1) It is easy to see that for any $n \in Z_{>0}$ there exists $M=M(n) \in Z_{>0}$ such that if $\left\{A^{i} \mid i \in I\right\}$ is. a family of ideals of bounded type $n$, then $\operatorname{dim} A^{i} \geqq r$ for all $i \in I$ if and only if there is a chain of proper prime ideals

$$
A^{i}=A_{0}^{i} \varsubsetneqq A_{1}^{i} \varsubsetneqq A_{2}^{i} \varsubsetneqq \cdots \varsubsetneqq A_{\tau}^{i}
$$

such that $\left\{A_{j}^{i} \mid i \in I\right\}$ is of bounded type $M$ for $j=0, \ldots, r$. The result then follows from 2.5 and 2.6 . 
(2) If $X^{*}$ is irreducible and contained in affine $n$-space, then $X^{*}$ is nonsingular $\Leftrightarrow$ rank of $\left\|\partial f / \partial X_{k}\right\|=n-\operatorname{dim} X^{*}$ at every point of $X^{*}$ (where $f$ ranges over a basis of the ideal $A^{*}$ of $\left.X^{*} ; k=1, \ldots, n\right)$. The right-hand side of $\Leftrightarrow$ can be seen to be a first-order predicate of the coefficients of the polynomials $f \in A^{*}$, and hence by the basic properties of ultraproducts and part (1), $X^{*}$ is nonsingular $\Leftrightarrow$ rank of $\left\|\partial f^{i} / \partial X_{k}\right\|=n-\operatorname{dim} X^{i}$ at every point of $X^{i}$ for almost all $i$ (w.r.t.D), (where $f^{i}$ ranges over a basis of the ideal $A^{i}$ of $\left.X^{i}\right) \Leftrightarrow X^{i}$ is nonsingular a.e. (w.r.t.D). If $X^{*}$ is not irreducible, we can write $A^{*}$ as an intersection of prime ideals and use 2.6 and the above argument to prove the result.

3. Ultraproducts of abstract varieties. Hironaka's Main Theorem I [2, p. 132], which we want to apply, is stated for reduced and irreducible algebraic $F$-schemes; but for our purposes it will be most convenient to view such schemes as abstract $F$-varieties in the sense of Weil, i.e. as a collection $\left[V_{j} ; \Phi_{j k}\right](j, k \in J)$ of affine varieties $V_{j}$ and a consistent set of coherent birational morphisms $\Phi_{j k}: V_{j} \rightarrow V_{k}$. (We extend the definition in Weil [12] to the nonabsolutely irreducible case.)

3.1 Definitions. An "embedded" abstract $F$-variety is an abstract $F$-variety $X=\left[V_{j} ; \Phi_{j k}\right](j, k \in J)$ together with embeddings of each of the $V_{j}$ into some affine space. $X$ is of bounded type $M$ if card $J \leqq M$, the $V_{j}$ are all of bounded type $M$, and the function field isomorphisms $\varphi_{j k}$ corresponding to the birational morphisms $\Phi_{j k}$ are of bounded type $M$ where, if $V_{j}$ (resp. $V_{k}$ ) has ideal $A_{j}$ (resp. $A_{k}$ ), $\varphi_{j k}$, mapping the quotient field of $F\left[Y_{1}, \ldots, Y_{n_{k}}\right] / A_{k}$ to the quotient field of $F\left[X_{12}, \ldots, X_{n}\right] / A_{j}$ is of bounded type $M$ if for each $l=1, \ldots, n_{k}$ there are polynomials $\alpha_{l}(X), \beta_{l}(X)$ of degree $\leqq M$ such that $\varphi_{j k}\left(Y_{l}+A_{k}\right)$ is represented by $\alpha_{l}(X) / \beta_{l}(X)$.

A family of "embedded" abstract $F^{i}$-varieties $X^{i}=\left[V_{j}^{i} ; \Phi_{j k}^{i}\right]$ of bounded type is defined in the (by now) obvious way. The ultraproduct (w.r.t.D) of the $X^{i}$ is then defined to be the collection $X^{*}=X_{D}^{*}=\left[V_{j}^{*} ; \Phi_{j k}^{*}\right]$ where $\Phi_{j k}^{*}$ corresponds to the field map $\varphi_{j k}^{*}$ which is defined by the ultraproducts $\alpha_{l}^{*}(X), \beta_{l}^{*}(X)$ of the polynomials $\alpha_{l}^{i}(X), \beta_{l}^{i}(X)$ which define $\varphi_{j k}^{i}$.

It may be checked that $\varphi_{j k}^{*}$ is an isomorphism if and only if the $\varphi_{j k}^{i}$ are isomorphisms for almost all $i$ (w.r.t.D). Hence $\varphi_{j k}^{*}$ does correspond to a birational morphism. In order for $X^{*}$ to be an abstract variety we must confirm that the $\Phi_{j k}^{*}$ are consistent and coherent. Consistency is easy, using the basic properties of ultraproducts and 2.4. As for coherency, fix $j$ and $k \in J$ and let $C_{j k}$ (resp. $C_{k j}$ ) be the ideal in $F^{*}\left[X_{1}, \ldots, X_{n}\right]$ (resp. $F^{*}\left[Y_{1}, \ldots, Y_{n_{k}}\right]$ ) defining the closed subset of $V_{j}^{*}$ (resp. $V_{k}^{*}$ ) where $\Phi_{j k}^{*}\left(\right.$ resp. $\Phi_{k j}^{*}$ ) is not regular, and let

$$
E_{j k} \subseteq F^{*}\left[X_{1}, \ldots, X_{n j}, Y_{1}, \ldots, Y_{n_{k}}\right]
$$

be the ideal of the graph of $\Phi_{j k}^{*}$; then $\Phi_{j k}^{*}$ is coherent if and only if

$$
E_{j k}+C_{j k} \cdot F^{*}[X, Y]=F^{*}[X, Y]=E_{j k}+C_{k j} \cdot F^{*}[X, Y]
$$


(see Weil [12, p. 178]). Let $C_{j k}^{i}, C_{k j}^{i}, E_{j k}^{i}$ be the corresponding ideals for $\Phi_{j k}^{i}$; then since we know $\Phi_{j k}^{i}$ is coherent for every $i \in I$, we know that the corresponding equation to $\left(^{*}\right)$ holds for all $i$. Hence the desired result follows from 2.4 and the following

3.2 THEOREM. There exists a set $S \in D$ such that the families of ideals $\left\{C_{j k}^{i} \mid i \in S\right\}$ and $\left\{E_{j k}^{i} \mid i \in S\right\}$ are of bounded type and their ultraproducts w.r.t.D equal $C_{j k}$ and $E_{j k}$ respectively.

Proof. The result for $C_{j k}$ is a consequence of the definitions (see [12, p. 178]) and the fact that if $\left\{A^{i} \mid i \in I\right\}$ and $\left\{B^{i} \mid i \in I\right\}$ are families of ideals of bounded type then $\left\{\left(A^{i}: B^{i}\right) \mid i \in I\right\}$ and $\left\{A^{i} \cap B^{i} \mid i \in I\right\}$ are of bounded type (see $\left.[1, \S 3]\right)$.

$E_{j k}$ is the kernel of the homomorphism

$$
\beta: F^{*}\left[X_{1}, \ldots, X_{n_{j}}, Y_{1}, \ldots, Y_{n_{k}}\right] \rightarrow F^{*}\left(x_{1}^{*}, \ldots, x_{n_{j}}^{*}\right)
$$

defined by $\beta\left(X_{u}\right)=x_{u}^{*}, \beta\left(Y_{v}\right)=\varphi_{j k}^{*}\left(Y_{v}+A_{k}^{*}\right)$, where

$$
F^{*}\left[x_{1}^{*}, \ldots, x_{n j}^{*}\right]=F^{*}\left[X_{1}, \ldots, X_{n}\right] / A_{j}^{*} .
$$

$E_{j k}^{i}$ is the kernel of the similarly defined homomorphism $\beta^{i}$ (i.e. $\beta^{i}\left(X_{\mu}\right)=x_{\mu}^{i}$, $\left.\beta^{i}\left(Y_{v}\right)=\varphi_{j k}^{i}\left(Y_{v}+A_{k}^{i}\right)\right)$. Now by choosing representatives of the coefficients of a set of generators of $E_{j k}$, define ideals $B_{j k}^{i} \subseteq F^{i}[X, Y]$ of bounded type such that $\left(B_{j k}^{i}\right)_{D}^{*}=E_{j k}$. If we prove that $B_{j k}^{i}=E_{j k}^{i}$ on a set of $D$ then we are done. Now $\beta\left(E_{j k}\right)=0$ implies $\beta^{i}\left(B_{j k}^{i}\right)=0$ a.e. and so $B_{j k}^{i} \subseteq E_{j k}^{i}$ a.e. Moreover $E_{j k}$ and $E_{j k}^{i}, i \in I$, are prime because they are the kernels of homomorphisms into fields and $B_{j k}^{i}$ is prime a.e. by 2.6 . Finally $\operatorname{dim} B_{j k}^{i}=\operatorname{dim} E_{j k}=\operatorname{dim} E_{j k}^{i}$ a.e. by 2.8 . Therefore we must have $B_{j k}^{i}=E_{j k}^{i}$ a.e.

REMARK. By a suitable choice of nonprincipal ultrafilter $D$ in 3.2 we can conclude that the families $\left\{C_{j k}^{i} \mid i \in I\right\}$ and $\left\{E_{j k}^{i} \mid i \in I\right\}$ are of bounded type.

3.3. In $\S 1$ a family $\left\{X^{i}\right\}$ of projective varieties was defined to be of bounded type if the family $\left\{A^{i} \subseteq F^{i}\left[X_{0}, \ldots, X_{n}^{i}\right]\right\}$ of their homogeneous ideals was of bounded type. Now a projective variety has a canonical representation as an abstract variety (the affine components are the complements of the hypersurfaces $X_{i}=0$ ); and it is easy to see that $\left\{X^{i}\right\}$ is of bounded type in the sense of $\S 1$ if and only if the corresponding family of abstract varieties is of bounded type in the sense of $\S 3$.

4. Ultraproducts of monoidal transformations. If $V$ is an affine variety with ideal $A \subseteq F\left[X_{1}, \ldots, X_{n}\right]$ and $I=\left\langle f_{1}, \ldots, f_{r}\right\rangle$ is an ideal with $A \subseteq I \subseteq F\left[X_{1}, \ldots, X_{n}\right]$, let $F\left[x_{1}, \ldots, x_{n}\right]=F\left[X_{1}, \ldots, X_{n}\right] / A$, and $R_{l}=F\left[x_{1}, \ldots, x_{n}\right]\left[f_{l}^{-1} I\right]=$ subring of $F\left[x_{1}, \ldots, x_{n}\right]_{f_{l}(x)}$ generated over $F\left[x_{1}, \ldots, x_{n}\right]$ by $f_{1}(x) / f_{l}(x), \ldots, f_{r}(x) / f_{l}(x)$; then the monoidal transformation of $V$ over $F$ with center $I$ is the abstract variety $\left[W_{l} ; \Psi_{l m}\right]$ $(1 \leqq l, m \leqq r)$ where $W_{l}=\operatorname{Spec}\left(R_{l}\right)$ and $\Psi_{l m}$ corresponds to the canonical isomorphism of the function fields of $W_{l}$ and $W_{m}\left(\cong F\left(x_{1}, \ldots, x_{n}\right)\right.$ ).

If $X=\left[V_{j} ; \Phi_{j k}\right](j, k \in J)$ is an "embedded" abstract $F$-variety and $A_{j}$ is the ideal of $V_{j}$, we regard a (quasi-coherent) ideal $\mathscr{I}$ on $X$ as a collection $\left\{I_{j}\right\}$ of polynomial 
ideals $I_{j}$ such that $A_{j} \subseteq I_{j}$ and the sheaves of ideals which the $I_{j}$ determine on the $V_{j}$ agree on the "overlaps" $V_{j} \cap V_{k}$. The monoidal transformation of $X$ over $F$ with center $\mathscr{I}$ is then the (abstract) variety $Y$ obtained by "patching" the monoidal transformations of the $V_{j}$ over $F$ with center $I_{j}$; there is a canonical projection $\pi: Y \rightarrow X$, defined over $F$.

4.1 TheOREM. Let $X^{i}, i \in I$, be "embedded" abstract varieties of bounded type and $X^{*}$ their ultraproduct with respect to $D$. Moreover for $i \in I$ let $\mathscr{I}^{i}=\left\{I_{j}^{i}\right\}$ be an ideal on $X^{i}$ such that the $I_{j}^{i}$ are of bounded type and such that $\left\{I_{j}^{*}\right\}$ is an ideal, $\mathscr{I}^{*}$, on $X^{*}$. Then if $Y^{i}$ is the monoidal transformation of $X^{i}$ with center $\mathscr{I}^{i}$, the affine components of the $Y^{i}$ may be embedded in affine space so that the $Y^{i}$ are of bounded type and their ultraproduct $Y^{*}$ (w.r.t.D) is the monoidal transformation of $X^{*}$ with center $\mathscr{I}^{*}$.

Proof. We may suppose $X^{i}$ is affine, say with ideal $A^{i} \subseteq F^{i}\left[X_{1}, \ldots, X_{n}\right]$, and suppose $\mathscr{I}^{i}=\left\langle f_{1}^{i}(X), \ldots, f_{r}^{i}(X)\right\rangle$. Then we can embed the affine components $W_{l}^{i}$ $(l=1, \ldots, r)$ of $Y^{i}$ so that the ideal of $W_{l}^{i}$ is the kernel of the homomorphism

$$
\alpha_{l}^{i}: F^{i}\left[X_{1}, \ldots, X_{n}, Y_{1}, \ldots, Y_{r}\right] \rightarrow F^{i}\left[X_{1}, \ldots, X_{n}, Z\right] /\left\langle A^{i}, 1-Z f_{l}^{i}(X)\right\rangle
$$

where $\alpha_{l}^{i}\left(X_{u}\right)=\bar{X}_{\mu}, \alpha_{l}^{i}\left(Y_{v}\right)=\operatorname{cl}\left(Z f_{v}^{i}(X)\right), \mu=1, \ldots, n ; \nu=1, \ldots, r$. The monoidal transformation of $X^{*}$ with center $\mathscr{I}^{*}$ is defined by an analogous set of maps

$$
\alpha_{l}^{*}: F^{*}\left[X_{1}, \ldots, X_{n}, Y_{1}, \ldots, Y_{\mathrm{r}}\right] \rightarrow F^{*}\left[X_{1}, \ldots, X_{n}, Z\right] /\left\langle A^{*}, 1-Z f_{l}^{*}(X)\right\rangle .
$$

The method of proof is then the same as in the proof of the second part of 3.2.

We are now ready to prove the main theorem:

4.2 TheOREM. For any positive integer $M$ there exists a finite set $P_{0}(M)$ of primes, and positive integers $t^{\prime}, M^{\prime}$, such that if $X$ is any "embedded" abstract $F$ variety satisfying:

(1) characteristic of $F$ is not in $P_{0}(M)$; and

(2) $X$ is of bounded type $M$;

then there exists a finite sequence of monoidal transformations of $F$-varieties $\pi_{l}: X_{l+1} \rightarrow X_{l}, 0 \leqq l<t$, such that $t \leqq t^{\prime}, X_{0}=X, X_{t}$ is nonsingular and for all $l<t$ :

(3) the center of $\pi_{l}$, say $Y_{l}$, is nonsingular;

(4) $Y_{l}$ does not contain any nonsingular point of $X_{l}$; and

(5) $X_{l}$ is of bounded type $M^{\prime}(l=0, \ldots, t)$.

Proof. We first exclude the case of characteristic zero. If the theorem is false, there exists an infinite subset $S=\left\{p_{n} \in P \mid n \in Z_{>0}\right\}$ of $P=$ set of primes, indexed by $Z_{>0}$, such that $p_{n} \neq p_{m}$ for $n \neq m$ and for each $p=p_{n} \in S$ there is a field $F^{p}$ of characteristic $p$ and an "embedded" abstract $F^{p}$-variety $X^{p}=\left[V_{j}^{p} ; \Phi_{j k}^{p}\right](j, k \in J)$ of bounded type $M$ such that there is no finite sequence of monoidal transformations $\pi_{l}: X_{l+1} \rightarrow X_{l}, 0 \leqq l<t$ satisfying: $t \leqq n ; X_{0}=X^{p} ; X_{t}$ is nonsingular; $X_{l}$ is of 
bounded type $n$ for all $l$; and (3) and (4). Choose a nonprincipal ultrafilter $D$ on $P$ such that $S \in D$. Let $F^{*}=\prod_{p \in P} F^{p} / D$ and let $X^{*}=$ the ultraproduct of the $X^{p}$, $p \in P$, with respect to $D$ (defined because $S \in D$ ). Then Char $F^{*}=0$, so by Hironaka's Theorem, there is a finite sequence of monoidal transformations $\pi_{l}: X_{l+1} \rightarrow X_{l}$, $0 \leqq l<t$ such that $X_{0}=X^{*}, X_{t}$ is nonsingular, and the $\pi_{l}$ satisfy (3) and (4). Suppose $\mathscr{I}_{0}=\{I\},(j \in J)$ is the quasi-coherent sheaf of ideals on $X_{0}=\left[V_{j}^{*} ; \Phi_{j k}^{*}\right](j \in J)$ which is the center of $\pi_{0}$. Choose polynomial ideals $I_{j}^{p}$ of bounded type such that $\left(I_{j}^{p}\right)_{D}^{*}=I_{j}$ for all $j \in J$. We assert that for almost all $p$ (w.r.t.D) the ideals $I_{j}^{p}$ determine a quasi-coherent sheaf of ideals on $X^{p}$. Let $U_{j k}^{p}$ (resp. $U_{k j}^{p}$ ) be the open subset of $V_{j}^{p}$ (resp. $V_{k}^{p}$ ) where $\Phi_{j k}^{p}$ (resp. $\Phi_{k j}^{p}$ ) is regular; then our assertion is that the sheaves on $V_{j}^{p}$ and $V_{k}^{p}$ determined by $I_{j}^{p}$ and $I_{k}^{p}$ respectively, when restricted to $U_{j k}^{p}$ and $U_{k j}^{p}$ respectively correspond under $\Phi_{j k}^{p}$. Let $U_{j k}$ (resp. $U_{k j}$ ) be the open subset of $V_{j}^{*}$ (resp. $V_{k}^{*}$ ) where $\Phi_{j k}^{*}$ (resp. $\Phi_{k j}^{*}$ ) is regular. We may choose polynomials $f_{1}, \ldots, f_{r} \in F^{*}\left[X_{1}, \ldots, X_{n_{j}}\right], g_{1}, \ldots, g_{r} \in F^{*}\left[Y_{1}, \ldots, Y_{n_{k}}\right]$ such that $\bigcup_{i=1}^{r}\left(V_{j}^{*}\right)_{f_{i}}=U_{j k}$, $\bigcup_{i=1}^{r}\left(V_{k}^{*}\right)_{g_{i}}=U_{k j}$, and such that $\Phi_{k j k}^{*}$ induces an isomorphism: $\left(V_{j}^{*}\right)_{f_{i}} \rightarrow\left(V_{k}^{*}\right)_{g_{i}}$, i.e. (if $A_{j}^{*}$ (resp. $A_{k}^{*}$ ) is the ideal of $V_{j}^{*}$ (resp. $\left.V_{k}^{*}\right)$ ) an isomorphism

$\psi_{j k}^{*}: F^{*}\left[X_{0}, X_{1}, \ldots, X_{n_{j}}\right] /\left\langle A_{j}^{*}, 1-f_{i}(X) X_{0}\right\rangle \rightarrow F^{*}\left[Y_{0}, Y_{1}, \ldots, Y_{n_{k}}\right] /\left\langle A_{k}^{*}, 1-g_{i}(Y) Y_{0}\right\rangle$

for each $i=1, \ldots, r$. Since $\mathscr{I}_{0}$ is an ideal, $\psi_{j k}^{*}$ takes the ideal $\left\langle I_{j}, 1-f_{i}(X) X_{0}\right\rangle$ onto $\left\langle I_{k}, 1-g_{i}(Y) Y_{0}\right\rangle$. Choosing polynomials $f_{1}^{p}, \ldots, f_{r}^{p}, g_{1}^{p}, \ldots, g_{r}^{p}$ over $F^{p}$ of bounded degree such that $f_{i}=\left(f_{i}^{p}\right)^{*}, g_{i}=\left(g_{i}^{p}\right)^{*}$, we conclude (using 3.2) that $\bigcup_{i=1}^{r}\left(V_{j}^{p}\right)_{f_{i}^{p}}=U_{j k}^{p}$ and $\bigcup_{i=1}^{r}\left(V_{k}^{p}\right)_{g_{i}^{p}}=U_{k j}^{p}$, and there exist isomorphisms $\psi_{j k}^{p}$ taking $\left\langle\dot{I}_{j}, 1-f_{i}^{p}(X) X_{0}\right\rangle$ onto $\left\langle I_{k}, 1-g_{i}^{p}(Y) Y_{0}\right\rangle$; thus $\mathscr{I}_{0}^{p}=\left\{I_{j}^{p}\right\}$ is a quasi-coherent ideal on $X_{0}^{p}$ a.e. (w.r.t.D).

Let $\pi_{0}^{p}: X_{1}^{p} \rightarrow X_{0}^{p}$ be the monoidal transformation of $X_{0}^{p}$ with center $\mathscr{I}_{0}^{p}$. By 4.1 we can assume the $X_{1}^{p}$ are "embedded" abstract $F^{p}$-varieties of bounded type such that $\left(X_{1}^{p}\right)_{D}^{*}=X_{1}$. We then repeat the procedure above for $\mathscr{I}_{1}=$ center of $\pi_{1}: X_{2} \rightarrow X_{1}$ and continue for $t$ steps. We get a set $T \in D$ and for each $p \in T$, a sequence $\pi_{l}^{p}: X_{l+1}^{p} \rightarrow X_{l}^{p}$, such that the $X_{l}^{p}$ are of bounded type and $X_{l}=\left(X_{l}^{p}\right)^{*}$ for $l=0, \ldots, t$; moreover by $2.8, X_{t}^{p}$ is nonsingular and (3) and (4) are satisfied for $p \in T$. Thus we have a contradiction of our original assumption (because $S \cap T$ is infinite).

To prove the existence of the bounds $t^{\prime}$ and $M^{\prime}$ in the case of varieties defined over fields of characteristic zero, we argue by contradiction as before: let $I=Z_{>0}$ and for each $n \in I$, suppose there is a $F^{n}$-variety $X^{n}\left(\right.$ Char $\left.F^{n}=0\right)$ which cannot be resolved by a sequence of length $\leqq n$ of monoidal transformations of bounded type $n$. Letting $D$ be a nonprincipal ultrafilter on $I$, take ultraproducts and argue as before.

4.3 Proof of Theorem A. The existence of $P_{0}$ is an immediate consequence of $4.2,3.3$, and 1.4 once we know that the existence of $M^{\prime}$ such that the $X_{l}$ are of bounded type $M^{\prime}$ as abstract $F$-varieties implies the existence of $n^{\prime}$ and $d^{\prime}$ such that the $X_{l}$ are embeddable in projective $n^{\prime}$-space as varieties of degree $\leqq d^{\prime}$. But this 
fact is provable by means of another proof by contradiction: take an ultraproduct of counterexamples and use the fact that any monoidal transformation of a projective variety is embeddable in some projective space.

\section{REFERENCES}

1. G. Hermann, Die Frage der endliche vielen Schritte in der Theorie der Polynomideale, Math. Ann. 95 (1926), 736-788.

2. H. Hironaka, Resolution of singularities of an algebraic variety over a field of characteristic zero, Ann. of Math. 79 (1964), 109-326.

3. S. Kleiman, "Les théorèmes de finitude pour le foncteur de Picard" in Séminaire de géométrie algébrique, Inst. Haûtes Études Sci. (to appear).

4. S. Kochen, Ultraproducts in the theory of models, Ann. of Math. 74 (1961), 221-261.

5. J. König, Einleitung in die allgemeine Theorie der algebraischen Gröszen, Teubner, Leipzig, 1903.

6. W. M. Lambert, Effectiveness, elementary definability and prime polynomial ideals, Doctoral Dissertation, University of California, Los Angeles, 1965.

7. S. Lang, Introduction to algebraic geometry, Interscience, New York, 1958.

8. E. Lluis, Sur l'immersion des variétés algébriques, Ann. of Math. 62 (1955), 120-127.

9. A. Robinson, Introduction to model theory and to the metamathematics of algebra, North-Holland, Amsterdam, 1963.

10. - Théorie métamathématique des idéaux, Collections de Logique Mathématique, Paris-Louvain, 1955.

11. B. L. van der Waerden, Einfiürung in die algebraische Geometrie, Dover, New York, 1945.

12. A. Weil, Foundations of algebraic geometry, rev. ed., Colloq. Publ., Vol. 29, Amer. Math. Soc., Providence, R. I., 1962.

13. $\mathrm{O}$. Zariski, The concept of a simple point of an abstract algebraic variety, Trans. Amer. Math. Soc. 62 (1947), 1-52.

YALE UNIVERSITY,

New Haven, Connecticut 